\title{
AVOIDING PACKET LOSS IN GATEWAY REALLOCATION IN MOBILE WIMAX NETWORKS
}

\author{
Kusha K R \\ Student, Department of Computer Science and Engineering, Jawaharlal Nehru National College of Engineering, \\ Shimoga, VTU, Karnataka, India, kusha.karur@gmail.com
}

\begin{abstract}
Gateway reallocation algorithms are proposed to increase the capacity of WiMAX networks to serve more users. But most of the proposed solutions don't consider the momentary packet loss occurring during the reallocation process. This momentary packet loss can also affect the application quality of service. In this work, I propose an efficient adaptive buffering strategy to avoid this packet loss in the network. Through simulation I prove that the approach is effective against momentary packet loss during the gateway reallocation process.
\end{abstract}

Keywords - security; sensor networks, denial of sleep attack $* * *$

\section{INTRODUCTION}

The IEEE 802.16-series standards [1], [2] are expected to provide broadband wireless access for a variety of multimedia services. Like other IEEE 802-series standards, IEEE 802.16 working group standardizes physical (PHY) layer and Medium Access Control (MAC) layer only. To build a complete system, higher layers are still necessary. One of the major objectives of WiMAX Forum [3], thus, is to develop and standardize the WiMAX Forum Network Architecture [4], [5], [6], [7], which is evolving into Internet Protocol (IP)-based wireless network. The architecture is depicted in Figure 1. In Figure 1, the Access Service Network (ASN) provides wireless radio access for WiMAX subscribers. It consists of one ASN Gateway (ASN GW) and many base stations (BSs). Each ASN is connected to Connectivity Service Network (CSN), which provides IP connectivity services. To support IP mobility, Mobile IP (MIP) 1 is adopted. The Home Agent (HA) of a Mobile Station (MS) is located in the CSN of the MS's Home Network Service Provider (H-NSP). ASN GW supports the Foreign Agent (FA) functionality. The WiMAX Forum has defined a two-tiered mobility management: ASN Anchored Mobility and CSN Anchored Mobility.

ASN Anchored Mobility refers to the procedures associated with the MS's movement between BSs, which may belong to the same or different ASN GWs. In ASN Anchored Mobility, the context of the designated MS is transferred from the previous BS to the new BS. Without performing CSN Anchored Mobility, ASN Anchored Mobility can minimize handover delay and packet loss. For example, an MS may perform intra-ASN handover (e.g., changing from Flow (1) to Flow (2) in Figure 1) while still attaching to the same ASN GW. In addition, an MS may perform inter-ASN handover (e.g., changing from Flow (2) to Flow (3) in Figure 1) where the ASN GW A is the traffic anchor point and responsible for ASN-CSN tunneling. That is, traffic is still sent to ASN GW A, which then further tunnels traffic to ASN GW B. In Flow (1) and Flow (2), the MS is called Serving MS of ASN GW A. In Flow (3), the MS is called Anchored MS of ASN GW A and handover MS of ASN GW B. In such case, the ASN GW A and ASN GW B are called anchored ASN GW and Serving ASN GW, respectively.

CSN Anchored Mobility refers to the process of changing the traffic anchor point and is independent of the MS's link layer handover [4]. It is also called ASN GW reallocation. For example, if CSN Anchored Mobility is not performed, when MS roams from ASN GW B to ASN GW C in Fig. 1, ASN GW A will tunnel traffic to ASN GW C. The MS is still served by two ASN GWs (ASN GW A and ASN GW C). As before mentioned discussion, the MS is called Anchored MS of ASN GW A. Later on, the ASN GW A may request the MS to carry out CSN Anchored Mobility, i.e., ASN GW reallocation. This may happen due to the heavy load of the ASN GW A [8], to reduce end-to-end latency, or for resource optimization purposes [4], [5]. After performing ASN GW reallocation, the traffic anchor point is changed to ASN GW C. The MS then is not served by ASN GW A. This is shown in Fig. 1 after changing from Flow (4) to Flow (5).

Although the two-tiered mobility management defined in WiMAX potentially can minimize handover delay and packet loss, it leads to another problem: When to perform ASN GW reallocation? The WiMAX standards, however, only define the procedures for ASN Anchored Mobility and CSN Anchored Mobility. The standards do not address when the Anchored MSs should perform ASN GW reallocation to relocate the traffic anchor point from the anchored ASN GW 
to the serving ASN GW. The problem is left for vendors and operators to develop their own proprietary solutions.

Besides, the problem is closely related to Admission Control (AC), which is widely used in wireless networks to ensure service quality and reduce network congestion by limiting the number of MSs served in the network. If the ASN GW reallocation can be performed before the system becomes overloaded, the system may be able to accommodate more MSs. Therefore, a well-designed AC algorithm should cooperate with the ASN GW reallocation algorithm.

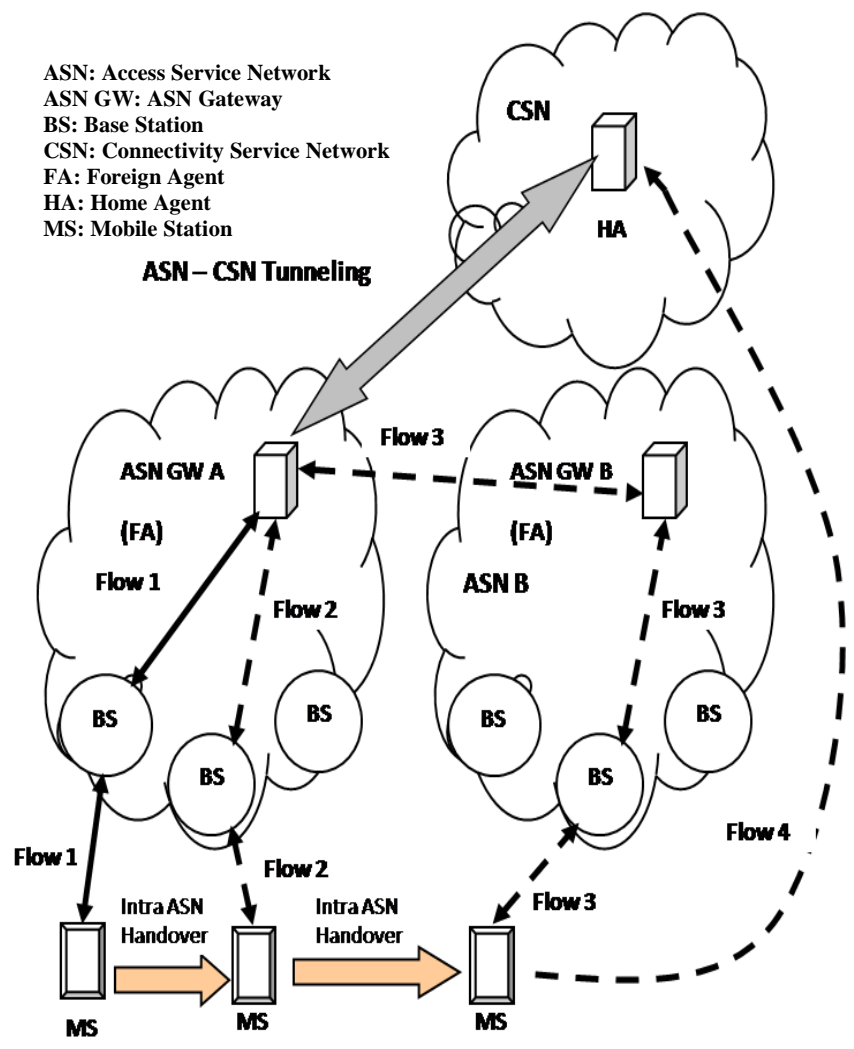

Figure 1: ASN Anchored Mobility and CSN Anchored Mobility in WiMAX networks.

The problem with the reallocation is that, typical reallocation on established session results in sudden packet loss during the handover procedure. Most solutions only focus on the reallocation process, but give little attention to the momentary packet loss during the reallocation process. I propose adaptive buffering solution to reduce this packet loss without affecting the quality of service.

\section{LITERATURE SURVEY}

In the literature survey, I explore current solutions to reduce packet loss in gateway reallocation process in Mobile WIMAX networks.
Zong-Hua Li and Jyh Cheng Chen in their work on gateway reallocation proposed a predictive mechanism to reduce the packet loss and handover latency. Their approach is based on predicting the connections to be reallocated and moving them in advance, so that capacity of network is increased and also there is less call drops because connections are available. But their approach does not consider momentary packet loss also they do reallocation on the connection which is loaded with packets causing momentary packet loss.

Ai-Chun Pang and Yi-Bing Lin in their work on approach for reallocation in UMTS network proposed to buffer packets at RNC to avoid packet loss. But their approach does not consider the case of multiple mobile stations downloading same contents. In this case buffering at RNC is not a viable solution. Also when the mobile station moves out of the area of RNC the packets queued at RNC is waste.

As see from related works mentioned, there is no optimized way to avoid this momentary packet loss, and I am the first to provide solution for same.

\section{OVERVIEW OF PROPOSED SOLUTION}

This proposed solution consists of two stages.

1. Buffering packets at CSN

2. Buffering packet at ASN.

Buffering packets at CSN is needed for the packets arriving from the internet side. Buffering at ASN is needed for the packets from the mobile station.

In the proposed solution, when a connection is to be reallocated based on the current rate of communication, buffer size need to be allocated is calculated and reserved in advance at the serving ASN and at the CSN. The packets arrived from mobile station is queued in the ASN buffer, till reallocation process is completed. The packets from internet is buffered at the gateway and delivered to the ASN once the gateway reallocation process is complete.

Buffering at ASN has a important advantage when compared to buffering at the RNC because even when the mobile station moves from one RNC area to another but in the same ASN.

\section{DETAILS OF PROPOSED SECURITY MECHANISM}

\section{A. Buffering at CSN}

At the gateway, buffer chunks are available. When a connection is to be reallocated, based on the rate of arrival of packets from internet the buffer chunks are allocated according to

Buffer Size $=$ Rate of Arrival of Packet $(\mathrm{RI}) \mathrm{x}$ Time to reallocate. 
The time to reallocate is most of cases constant, but when the number of reallocates is more this value will increase.

The packets arriving from internet are queued in the Buffer and provided to the connection on successful reallocation. In case of failure of reallocation the connection will be torn down and buffer space allocated is added back to the pool. The buffer space allocated is based on the current rate of arrival and thus the allocation process is very adaptive. Also if the reallocation is known a priori, the rate of packet arrival at internet can be reduced, there by the buffer size allocated is less.

\section{B. Buffering at ASN}

At ASN buffer space is allocated for the packets arriving from the mobile station. Based on the rate of arrival of packets from the mobile station, the buffer space is allocated according to the relation

Buffer Size $=$ Rate of Arrival of Packet $(\mathrm{Rm}) \times$ Time to reallocate

The packets from the mobile station is queued at the ASN and delivered to the CSN on establishment of the connection. The rate of arrival of packets from mobile station is reduced on the start of reallocation process and brought back to original level after the reallocation process is complete.

\section{Prediction Algorithm Changes For Reducing}

\section{Packet Loss}

Wiener prediction process proposed by Zhong Hua Li can be used for finding the connection to be reallocated. But in their original work the connection to be reallocated is found by estimating the expected serving rate of each ASN and reallocating the connection based on it. But I propose a further extension to this method, based on the expected serving rate the number of connection to be reallocated is found. The connections are then sorted in ascending order of the rate of packet transfer. The connection with low rate of packet transfer is first reallocated before connection with high rate of data transfer. This is done purposely to avoid packet loss; the best time for reallocation of connection is at the time of low data packet transfer rate. The previous solutions have not focused on this part, so there was momentary packet loss, but in my solution I have given more priority to connection with low data rate transfer and reallocated them.

\section{Buffer Space De-Allocation}

The buffer space is de allocated in following ways.

- In case of reallocation failure the connection is freed totally and buffer is freed back to pool.

- In case of reallocation success the packets in the buffer are cleared at the rate of packet transfer and once all packets are delivered, the buffer space is freed back to pool

\section{PERFORMANCE ANALYSIS}

I implemented the simulation using NS2 simulator and also simulated a WIMAX of $4 \mathrm{ASN}$ and 20 to 50 mobile in each ASN area and random movement of mobile nodes across the 4 ASN. The number of allowed connection between ASN and CSN is set as 10 and simulation is conducted.

I measure the number of packet loss during the reallocation time using this proposed solution and the WP based method. The results show that proposed solution has less packet loss compared with WP based solution. This reduced packet loss is due to the buffering of packets.

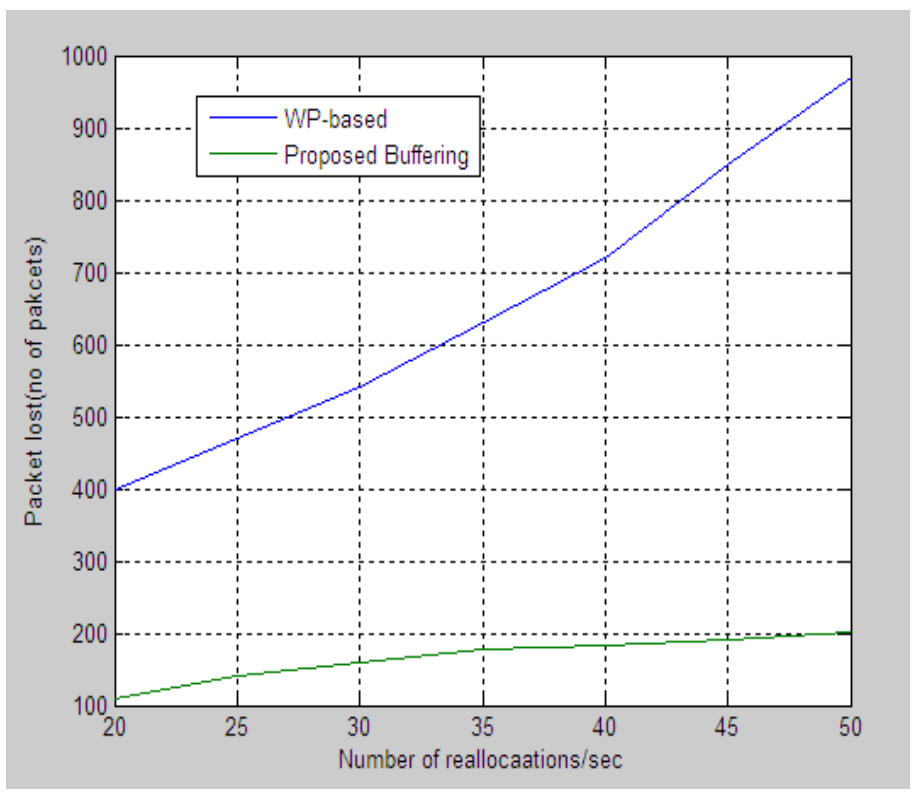

Figure 2: Number of reallocations per second versus Packets Lost in WP-based and proposed buffering.

\section{CONCLUSION AND ENHANCEMENTS}

In this paper, I have detailed buffering solution to avoid packet loss. Through extensive simulation I proved that that packet loss is reduced in this approach compared to previous solutions of buffering at RNC. The solution is very effective by giving more priority to low data rate connections to be reallocated faster compared to connections with higher data rate.

Not all kind of applications need mechanism for packet loss tolerance. Applications like VOIP can tolerate packet loss. In future I want to provide the buffering strategy based on the kind of application. 


\section{REFERENCES}

[1] IEEE 802.16-2004 Std., Air Interface for Fixed Broadband Wireless Access Systems, IEEE, Oct. 2004.

[2] IEEE 802.16e-2005 Std., Part 16: Air Interface for Fixed and Mobile Broadband Wireless Access SystemsAmendment 2: Physical and Medium Access Control Layers for Combined Fixed and Mobile WiMAX Forum, http://www.wimaxforum.org, 2011.

[3] WiMAX Forum Std. 1.0, Rev. 4, WiMAX Forum Network Architecture (Stage 2: Architecture Tenets, Reference Model and Reference Points), WiMAX, Feb. 2009.

[4] WiMAX forum Std. 1.0, Rev. 4, WiMAX Forum Network Architecture (Stage 3: Detailed Protocols and Procedures), WiMAX, Feb. 2009.

[5] L. Nuaymi, WiMAX: Technology for Broadband Wireless Access John Wiley, 2007.

[6] K. Etemad, "Overview of Mobile WiMAX Technology and Evolution," IEEE Comm. Mag., vol. 46, no. 10, pp. 31-40, Oct. 2008.

[7] Z.-H. Liu, S.-Y. Pan, and J.-C. Chen, "Access Service Network (ASN) Gateway Relocation Algorithms in WiMAX Networks," Proc. IEEE Int'l Conf. Comm. (ICC '08), pp. 2674-2679, May 2008.

[8] Y. Fang and Y. Zhang, "Call Admission Control Schemes and Performance Analysis in Wireless Mobile Networks," IEEE Trans. Vehicular Technology, vol. 51, no. 2, pp. 371-382, Mar. 2002. 